\title{
I mpacto Económico del dengue en el Instituto de Previsión Social: epidemia diciembre 2006 - julio 2007
}

\author{
Laura Flores', Edgar Giménez Caballero'", Sebastián Díaz Duba', J udith Torales' \\ I. Hospital de Clínicas, Universidad Nacional de Asunción \\ II. Instituto Desarrollo
}

Cómo referenciar este artículo/ How to reference this article:
Flores L, Giménez Caballero E, Díaz Duba S, Torales J. Impacto económico del dengue en el Instituto de Previsión Social: epidemia diciembre 2006 - julio 2007. Mem. Inst. Investig. Cienc. Salud. 2015; 13(2):78-85

\section{R E S U M E N}

El dengue es la enfermedad viral transmitida por vectores más extendida en el mundo, siendo una carga económica significativa en los países endémicos. Aunque se ha tratado de determinar el costo relacionado con los casos de dengue y su impacto económico en varios países, no se conoce la magnitud de su repercusión económica en nuestro país. El objetivo del estudio fue determinar los costos directos e indirectos asociados con la atención de dengue en el Instituto de Previsión Social en el 2007. Diseño observacional descriptivo de corte transverso. Se incluyeron todos los pacientes con diagnóstico de dengue, se calcularon: costos de insumos, hospitalización, análisis químicos y serología. Para los costos indirectos se consideró los subsidios pagados al personal y el salario mínimo diario del 2007. Se registraron 9819 consultas por síndrome febril, de los cuales 5960 casos fueron confirmados para dengue y 1447 hospitalizados. El costo día de insumos fue 38.180 Gs (7,6 US\$). El costo día laboratorio fue 17.660 Gs. Los costos de contratación de médicos para la contingencia fue 600.000 Gs día (120 US\$). El costo paciente/día internado 655.840 (131,1 US\$). El costo de atención ambulatoria fue 1.824.468G (364,8 US\$) por 7 días de tratamiento. Los costos indirectos fueron 358.701.798 G (71.740,3 US\$) por 2024 subsidios por dengue, correspondiente a 12.552 días de reposo. Los costos en la urgencia y ambulatorio representan más del $86 \%$, los costos a internados representa el 7\%, y los debidos al ausentismo laboral causado por la hospitalización y la convalecencia tras la enfermedad, representan el 7\%.

Palabras clave: Dengue, impacto económico, Paraguay.

\section{Economic I mpact of dengue fever in the Social Security Institute: December, 2006 - July 2007, epidemic}

\section{A B S T R A C T}

Dengue is a viral disease transmitted by vectors most widespread in the world, with a significant economic burden in endemic countries. Although it has been tried to determine the related cases of dengue and its economic impact in many countries cost the magnitude of its economic impact is not known in our country. The aim of the study was to determine the direct and indirect costs associated with the care of dengue in the Social Security Institute in 2007. observational transverse cut descriptive design. All patients with a diagnosis of dengue were calculated: input costs, hospitalization, chemical analysis and serology. Indirect costs to the allowances paid to staff and the daily minimum wage of 2007. 9819 consultations were recorded by febrile syndrome, of which 5960 cases were confirmed hospitalized for dengue and 1447 were considered. The day was $38.180 \mathrm{G}$ cost of inputs (7.6 USS). The cost was $17.660 \mathrm{G}$ day laboratory.

Fecha de recepción: agosto 2014. Fecha de aceptación: agosto 2015.

Autor correspondiente: Laura Flores. Hospital de Clínicas, UNA.

E-mail: floreslaurapy@yahoo.com 
The costs of hiring doctors for the contingency was 600.000G day (120 USS). The patient / day boarding cost 655840 (131.1 USS). The cost of outpatient care was 1.824.468G (364.8 USS) for 7 days of treatment. Indirect costs were $358701798 \mathrm{G}$ (71740.3 USS) 2024 dengue benefits corresponding to 12,552 days of rest. The costs in the emergency and ambulatory represent more than $86 \%$, the boarding costs up $7 \%$, and due to absenteeism caused by hospitalization and convalescence after illness, accounting for $7 \%$.

Keywords: Dengue, economic impact, Paraguay.

\section{NTRODUCCIÓN}

El Dengue es la enfermedad viral trasmitida por vectores más extendida en el mundo. Constituye uno de los mayores retos en salud pública de este siglo (1). Es producida por un Flavivirus (familia Flaviviradae) transmitida por el mosquito Aedes aegypti. Se conocen cuatro serotipos relacionados entre sí antigénicamente (DEN-1 al DEN-4) los cuales pueden causar diversas formas clínicas $(2,3)$.

En las Américas, el número de casos ha aumentado sostenidamente durante los últimos 25 años y este aumento se interpreta como una falla de las políticas de Salud Pública (4).

En el período de 1970 a 1998, el número de casos de dengue se cuadruplicó hasta alcanzar la cifra máxima de 1,3 millones, con más de 3.600 muertes. Actualmente el dengue es endémico en más de 100 países del sudeste asiático, el Pacífico occidental, América, África y el Medio Oriente.

Aunque no se ha determinado con exactitud la carga real de la enfermedad, se estima que de los 2500 millones de personas que viven en áreas de riesgo de transmisión, 50 millones se infectan anualmente y más de 500000 contraen su forma más grave. Durante las epidemias, las tasas de ataque pueden llegar a afectar a $80-90 \%$ de las personas susceptibles (5) y la letalidad puede ser mayor de $5 \%(6,7)$.

A pesar de las estrategias desarrolladas de prevención y control, la introducción de nuevos serotipos, así como la circulación de cepas más virulentas en poblaciones que cuentan con un número considerable de personas susceptibles con una infestación moderada o elevada con el mosquito Aedes aegypti y la falta de reconocimiento por parte del personal de salud de los casos graves apunta a prolongar esta epidemia y con ella los casos mortales (5).

Tan importante como evitar la transmisión del dengue es la preparación de los sistemas de salud para atender adecuadamente a los enfermos y evitar su muerte. Se debe aspirar a no tener epidemias, pero, si ocurren, se debe lograr que no haya fallecidos. Un buen administrador de salud es capaz de salvar más vidas durante una epidemia de dengue que los médicos e intensivistas ${ }^{1}$. Esto se debe a que el tratamiento individual de cada enfermo no puede aplicarse adecuadamente si no está enmarcado en un conjunto de medidas organizativas y de capacitación que deben ejecutarse en cada hospital o centro de atención primaria.

El impacto económico de las epidemias de dengue no se ha estudiado a fondo. Países como Cuba, Puerto Rico y Tailandia informan gastos de US\$ 6,8 a 103 US\$ millones solo en atención médica, medidas para el control vectorial, horas de trabajo perdidas y pérdidas por la disminución del turismo. En Cuba en 1.981, el costo aproximado por enfermo fue de 299 US\$, en Puerto Rico en 1.977 se estimó entre 26 y 31 US\$ (8-10). Se estima que en el período de 1.984 a 1.994 se perdieron 658 años de vida en Puerto Rico pérdida similar a la informada en otras partes del mundo en conexión con esta enfermedad y otros graves problemas de salud, como el paludismo y la tuberculosis (11).

Un estudio en el estado de Zulia en Venezuela, en el periodo de enero de 1.997 a 
diciembre de 2003 reveló un costo de US\$94 por paciente por costo de hospitalización, en total los costos directos ascendieron a US\$474,251.7 y los costos indirectos a 873.825.84 US\$ (12).

La primera y extensa epidemia de Dengue registrada en Paraguay ocurrió entre 1988 y 1989, con circulación de DEN-1 y un total de 41.990 casos en todo el territorio. Desde 1989 hasta noviembre de 1998, oficialmente no se reportaron casos (13). En el 2.000, se registraron 24.282 casos de Dengue del serotipo 1 (14). En diciembre de 2.006 a junio de 2007 se registra la Tercera Epidemia con 28.182 casos del serotipo 3 (15).

En el Instituto de Previsión Social (IPS) se registraron 10.000 casos sospechosos de dengue; desde diciembre de 2006 a junio de 2007 con un caso de $\mathrm{DH}$ (16); este centro asegura a la mayor fuerza laboral de nuestro país que representa al sector productivo.

No se tienen datos sobre el impacto económico de esta patología en nuestro país, tanto a nivel de gastos por hospitalización, como de pérdidas de días laborales; por lo cual el objetivo del presente trabajo es determinar los costos directos e indirectos asociados con la atención de casos de dengue en el Instituto de Previsión Social durante el periodo de tiempo de diciembre de 2006 a julio de 2007.

\section{MATERI ALES Y MÉTODO}

Para este estudio observacional, descriptivo de corte transverso se incluyeron todos los pacientes que consultaron en los servicios del Instituto de Previsión Social con diagnóstico clínico y/o serológico de dengue en el período de diciembre de 2006 a julio de 2007, se incluyeron aquellos que recibieron tratamiento en forma ambulatoria o hayan sido internados.

Fueron excluidos aquellos pacientes que no tenían la ficha epidemiológica llenada correctamente y aquellos que se negaron al tratamiento ambulatorio, o a la internación.

Para el reclutamiento se tomaron los datos del departamento de epidemiología del IPS, donde figuran las fichas epidemiológicas de los casos, se tomaron el total de casos de dengue, los que recibieron tratamiento ambulatorio y los que requirieron internación en sala y/o terapia intensiva.

Costos directos: Para calcular el costo de hospitalización mensurable relacionado con los insumos y los servicios utilizados con la atención médica hospitalaria de estos pacientes, se tomaron en cuenta el costo de los medicamentos, las soluciones de hidratación parenteral y los insumos desechables según datos del 2007, con esto se calculó el costo día-cama por paciente. Además del costo día-cama se agregaron los gastos de laboratorio clínico, calculados a partir del costo de los exámenes realizados más frecuentemente a los pacientes con dengue, que son la hemoglobina, hematocrito, recuento de glóbulos blancos y fórmula leucocitaria, recuento de plaquetas, y las transaminasas. Se calcularon también los costos de contratación de médicos para cubrir la contingencia del dengue. Para los pacientes internados se consideró una estancia media de 4 días según datos proporcionados por los Departamentos de Epidemiología, Emergencias y Medicina Interna y datos de autores nacionales $(17,18)$. Para los pacientes ambulatorios se consideró los costos de tratamiento administrados de acuerdo a los protocolos de dengue.

Costos Indirectos: Las pérdidas relacionadas con el ausentismo laboral durante la hospitalización y la convalecencia de los pacientes con dengue fueron proporcionadas por el Departamento de Medicina Laboral del IPS, quien procesa los beneficios a corto plazo por enfermedad común y profesional. Estos costos se calcularon de acuerdo en función de la proporción de la población empleada en el país, se utilizó el salario mínimo diario correspondiente al año 2007. Se consideró que los pacientes con dengue pierden en promedio siete días laborales, los pacientes con Dengue hemorrágico 14 días laborales $(12,19,20)$. 


\section{Consideraciones éticas}

Se solicitó permiso a las autoridades del departamento de Epidemiología, al Laboratorio Central y al departamento de Compras e Insumos del Instituto de Previsión Social para la revisión de los datos. También se solicitó permiso a los Jefes de las Unidades de Medicina Interna y de Terapia Intensiva de dicho nosocomio, a la Dirección de Medicina Preventiva y al Departamento de Medicina Laboral, quienes proporcionaron los datos.

\section{RESULTADOS}

Se registraron 9819 consultas por síndrome febril en el periodo estudiado. Fueron confirmados 5960 casos según criterios clínicos, por nexo epidemiológico y/o laboratorial.

De los 5960 casos, requirieron internación 1447 pacientes, 489 en el Hospital Central (HC) y 958 en las clínicas periféricas e interior del país. De los 489 internados en HC, 344 fueron adultos (70\%) y $145(30 \%)$ niños. Tres casos fueron admitidos en la Unidad de Terapia Intensiva de adultos; la letalidad asociado a comorbilidad fue de $0,05 \%$.

De los 344 adultos hospitalizados en el Hospital Central, 128 (37\%) eran de sexo femenino y 216 (63\%) del masculino. En el periodo de tiempo estudiado se internaron entre 7 y $25 \%$ de los que consultaron por dengue.

\section{Costos Directos}

Costo día-cama-paciente. Se estimaron para 4 días de internación. El costo diario por insumos fue de 38.180G (7,6 USS) y por cuatro días 131.620G (26,3 USS) (Tabla 1)

Costo día por estudios laboratoriales. El costo por estudios laboratoriales fue de 17.660 guaraníes por día y por cuatro días 31.670 G (Tabla 1).

Costo de contratación de médicos para la contingencia del dengue: 20 médicos por 900.000 por tres meses: 54.000.000 guaraníes.

Costo de contratación de médicos para la contingencia del dengue por día: 600.000 guaraníes (120 USS).

\section{Costo directo total}

Para los costos directos totales se tuvieron en cuenta los costos de insumos, de laboratorio y de contratación de médicos, Costo paciente día hospitalizado: 655.840 guaraníes (131,1 USS) (Tabla 2), todos los pacientes del HC por día: G 225.608.960 (45.121,7 USS); y todos los pacientes incluidos periféricas e interior G 949.000.480 (189.800, USS) (Tabla 3).

\section{Costos de atención ambulatoria}

Se estimaron para 7 días de tratamiento, medicación, laboratorio y honorarios médicos, G 1.824 .468 (364,8 USS) por cada paciente atendido y por todos G 10.873.829.280 (2.174.765 USS) (Tabla 4). 
Tabla 1. Costo directo diario y por 4 días por paciente

\begin{tabular}{lll}
\hline I TEMS & Por día (Gs.) & Por $\mathbf{4}$ días (Gs.) \\
\hline I nsumos & & \\
Hidratación (Suero fisiológico) & 26.200 & 104.800 \\
Descartables & 9.050 & 22.390 \\
Medicación & 2.930 & 4.430 \\
\hline Total insumos & $\mathbf{3 8 1 8 0}(\mathbf{7 , 6}$ USS) & $\mathbf{1 3 1 . 6 2 0 ~ ( 2 6 , 3 ~ U S S )}$ \\
\hline Estudios laboratoriales & & \\
Hemograma & 1890 & 7560 \\
Perfil hepático & 3080 & 6160 \\
TP & 2930 & 2930 \\
Urea & 770 & 1540 \\
Creatinina & 770 & 1540 \\
Glicemia & 770 & 1540 \\
Electrólitos & 2950 & 5900 \\
Gasometría & 4500 & 4500 \\
Serología para dengue & $\ldots \ldots .$. & $\ldots \ldots \ldots$ \\
\hline Total estudios laboratoriales & $\mathbf{1 7 . 6 6 0}(\mathbf{3 , 5}$ USS) & $\mathbf{3 1 . 6 7 0 ~ ( 6 , 3 ~ U S S ) ~}$ \\
\hline
\end{tabular}

Tabla 2. Costo Directo Total/día y por 4/días por paciente

\begin{tabular}{lclc}
\hline I TEMS & Por & día (Gs.) & Por 4 días (Gs.) \\
\hline Insumos & 38.180 & (7,6 USS) & $131620 \quad(26,3$ USS) \\
Laboratorio & 17.660 & $(3,5$ USS $)$ & $31670 \quad(6,3$ USS) \\
Honorarios & 600.000 & $(120$ USS $)$ & $2.400 .000 \quad(480$ USS $)$ \\
\hline Total & 655.840 & $(131,1$ USS $)$ & $2.563 .290 \quad(512,6$ USS) \\
\hline
\end{tabular}

Tabla 3. Costo de pacientes internados

\begin{tabular}{lll}
\hline $\mathbf{n}$ & Adultos HC/ día & Adultos HC/ 4 días \\
\hline 344 & $225.608 .960 \quad(45.121,7$ USS $)$ & $881.771 .760 \quad$ (176.354,3 USS) \\
& Todos los Internados/día & 4 días \\
1447 & 949.000 .480 (189.800,1 USS) & 3.709 .080 .630 (741.816,1 USS) \\
\hline
\end{tabular}


Tabla 4. Costos de atención ambulatoria por paciente y el total de pacientes

\begin{tabular}{lll}
\hline ITEMS & \multicolumn{2}{l}{ Tratamiento $\mathbf{7}$ días (Gs.) } \\
\hline Medicación & 4.938 & ( 1 USS $)$ \\
Laboratorio & 19.530 & ( 3,9 USS $)$ \\
Honorarios & 1.800 .000 & (360 USS) \\
\hline Total por paciente & 1.824 .468 & ( 364,8 USS) \\
\hline Total casos 5.960 & 10.873 .829 .280 & $(2.174 .765$ USS $)$ \\
\hline
\end{tabular}

\section{Costos indirectos}

Subsidios pagados por el IPS en concepto de dengue en el periodo de estudio.

Se abonaron en total por 2024 reposos, un total de 358.701.798 (71.740,3 USS), correspondientes a 12.552 días no trabajados con una media de 6,2 días de reposo (Tabla 5).

Tabla 5. Costos I ndirectos

\begin{tabular}{|c|c|c|c|}
\hline & $\mathrm{n}$ & Total días & Monto \\
\hline Subsidios * & 2024 & 12.552 (media: 6,2 días) & 358.701 .798 ( $71.740,3$ USS) \\
\hline Pérdida Laboral & 2024 & 12.552 & 829.624 .276 (165.924,8 \\
\hline Todos los reposos** & 5960 & 41.720 (media: 7 días) & $\begin{array}{l}2.313 .165 .400(462.633,1 \\
\text { USS) }\end{array}$ \\
\hline
\end{tabular}

Los subsidios se pagan a partir del segundo día de incapacidad, el monto corresponde al $50 \%$ del salario del trabajador de los últimos 4 meses, de acuerdo a la Ley Orgánica del Instituto de Previsión Social.

**Para los cálculos totales se consideró el salario mínimo del 2007 de 1.219.795 Gs.(55.445 Gs. el jornal diario)

\section{Total de costos}

Para el costo total se estimaron por 4 días de internación el total de casos de adultos del HC, más el total de tratamiento ambulatorio más el ausentismo laboral: 12.585.225.316 Gs (2.517.045, 1 USS) (Tabla 6).

Tabla 6. Total de costos

\begin{tabular}{lll}
\hline & Costos totales \\
\hline Paciente Internado (4 días) & 881.771 .760 Gs $\quad(176.354,3$ USS $)$ \\
Ambulatorio (7 días) & 10.873 .829 .280 Gs $\quad(2.174 .765,1$ USS $)$ \\
Ausentismo laboral & 829.624 .276 Gs $\quad(165.924,8$ USS $)$ \\
TOTAL & 12.585 .225 .316 Gs $\quad(2.517 .045,1$ USS $)$ \\
\hline
\end{tabular}




\section{DISCUSIÓN}

Es el primer trabajo en referencia al impacto económico de las epidemias del dengue en nuestro país. El diseño está hecho para la seguridad social, pero es extrapolable al sistema de salud pública y privada.

En este trabajo no se consideraron lo costos del control vectorial y químico, ya que la Seguridad Social no realiza estos controles. En algunas series estos costos representan cerca del 70 a $80 \%$ de los costos (10).

Tampoco se consideraron los costos del transporte, ni de alimentación como en los estudios de Armien y col en Panamá (21).

La conclusión de la mayoría de los estudios realizados sobre los costos de atención del dengue refleja que la atención médica de los pacientes con dengue está lejos de ser barata (22). Los costos de la atención a pacientes en la urgencia y ambulatorio representan más del $86 \%$, los costos de la atención a internados representa el 7\%, y los debidos al ausentismo laboral causado por la hospitalización y la convalecencia tras la enfermedad, representan el $7 \%$.

La tasa de letalidad durante el período analizado fue baja, menos del 0,1\%, similar a la letalidad en América latina, Valdés et al (12) encontraron tasas de letalidad de 5,8 por cada 100 casos de durante una epidemia de dengue (causada por el serotipo DEN-2) en Santiago de Cuba, lo cual contrasta con las cifras encontradas en este estudio.

EI costo de 131 USS por paciente día internado, contrasta otros estudios como el de Zulia (Venezuela) de 91 USS por paciente, el de Cuba de 42 USS por paciente, y similar al de Nicaragua donde se estimó 130 USS por paciente en 1994 (12), también debido a DEN 3.

En otro estudio hecho en el 2005 en cinco países de Latinoamérica (Brasil, Guatemala, El Salvador, Panamá y Venezuela) y tres de Asia (Camboya, Malasia y Tailandia), se encontró que los costos directos e indirectos de la atención de dengue del periodo 20012004 ascendían a U\$ 1,8 billones (22)

La gran variabilidad observada en el costo por la atención hospitalaria puede deberse a que el valor de los insumos y servicios en cada país depende de variables económicas intrínsecas, a los elementos considerados para el cálculo, y si el paciente es atendido en sistemas públicos, privados o de seguridad.

Se ha cestimado que por cada caso notificado de dengue, hay 10 enfermos menores de 15 años y 27 de más de 15 años que no se notificaron. Este subregistro suele deberse a que los enfermos no acuden a los centros médicos o a que no se diagnostican como casos de dengue, o que hayan acudido al sistema público o privado, por lo que el costo de esta enfermedad puede ser mucho mayor al presentado en este trabajo (11).

Dado que la precisión en el cálculo de los gastos generados por una enfermedad depende en gran medida de la información epidemiológica disponible y esta es insuficiente o inexacta en algunos casos, el Banco Mundial desarrolló un índice compuesto no monetario denominado "discapacidad ajustada por años de vida" para medir el impacto de la morbilidad y mortalidad en la población. Este indicador ha demostrado que el dengue tuvo un gran impacto en Puerto Rico y Myanmar, tanto en períodos epidémicos como interepidémicos, especialmente por sus costos indirectos relacionados con el ausentismo escolar y laboral debidos a la enfermedad. Otro indicador usado en algunos estudios es el QALY (calidad de vida $(11,12)$.

No se ha estimado el costo psicosocial/psicológico, stress emocional tanto de los trabajadores de salud como de la población, que ocasiona esta enfermedad, estos impactos debieran de tenerse en cuenta para futuras investigaciones.

El dengue constituye un importante problema de salud pública que ocasiona grandes gastos tanto en costos de atención, ausentismo laboral, esto afecta considerablemente al desarrollo de la economía regional y nacional.

Hay mucha literatura dispersa en referencia a la carga de la enfermedad, es necesaria 
y urgente, informaciones para los tomadores de decisiones para el control y prevención de la enfermedad, incluyendo la no tan lejana vacuna y el control vectorial (21).

\section{REFERENCI AS BI BLI OGRÁFI CAS}

1. Torres EM. La prevención de la mortalidad por dengue: un espacio y un reto para la atención primaria de salud. Rev Panam Salud Pública. 2006;20(1):60-74.

2. Mairuhu ATA, Wagenaar J, Brandjes DPM, van Gorp ECM. Dengue: an arthropod-borne disease of global importante. Eur J Clín Microbiol Infect Dis. 2004;23:425-33.

3. Gubler DJ, Clark GG. Dengue/dengue hemorrhagic fever: the emergence of a global health problem. Emerg Infect Dis. 1995; 1(2): 55-7.

4. Gubler D. The emergent of epidemic dengue fever and dengue hemorragia fever in the Americas: a case of failed public health policy. Rev Panam Salud Publica. 2005; 17 (4): 221-4.

5. Calisher $\mathrm{CH}$. Persistent emergence of dengue. Emerg Infect Dis. 2005;11(5):7389.

6. Guzmán MG, Kourí G. Dengue: an update. Lancet Infect Dis. 2002; 2(1):33-42.

7. Gubler DJ. The changing epidemiology of yellow fever and dengue, 1900 to 2003: Full circle? Comp Immunol Microbiol Infect Dis. 2004;27(5): 319-30.

8. Guzmán MG, Kourí G, Bravo J, Triana C. Estimación de las afectaciones económicas causadas como consecuencia de la epidemia de dengue hemorrágico ocurrida en Cuba en 1981. Rev Cubana Med Trop. 1992; 44(1): 13 $-7$.

9. Von Allmen SD, López-Correa RH, Woodall JP, Morens DM, Chiriboga J, Casta-Véllez A. Epidemic dengue fever in Puerto Rico, 1977: a cost analysis. Am J Trop Med Hyg. 1979; 28(6): 1040- 4.

10.Valdéz L, Vila J, Guzmán MG. Impacto economico de la epidemia de dengue 2 en Santiago de Cuba, 1997. Rev Cubana Méd Trop. 2002; 54:220-7.

11. Melzert MI, Rigau-Pérez JG, Clark GG, Reiter P, Gubler DJ. Using disability adjusted life years to assess the economic impact of dengue in Puerto Rico: 1984-1994. Am J Trop Med Hyg. 1998; 59(2): 265-71.

12. Añez G, Balza R, Valero N, Larreal $\mathrm{Y}$. Impacto económico del dengue y del dengue hemorrágico en el Estado de Zulia, Venezuela, 1997 - 2003. Rev Panam Salud Pública. 2006; 19(5):314-20.
13. Ministerio de Salud Pública y Bienestar Social, Dirección de Vigilancia de la Salud. Historia del dengue en Paraguay. Asunción : Ministerio de Salud Pública y Bienestar Social. [accedido 6 Set. 2014]. Disponible en: http://www. vigisalud.gov.py/index.php? option $=$ com_content $\&$ view $=$ article $\& i d=445 \&$ catid $=85 \&$ l témid $=151$.

14.Pan American Health Organization. World Health Organization. 2000: Number of reported cases of dengue \& dengue hemorrhagic fever (DHF), Region of the Americas (by country). Pan American Health Organization /citado 6 Set. 2014/. Disponible en: http://www2. paho.org/hq/

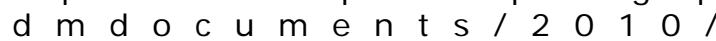
dengue_regional_data_2000.pdf.

15. Pan American Health Organization. Number of reported cases of dengue \& dengue hemorrhagic fever (DHF), in the Americas, by country: figures for 2007 (to week noted by each country). Pan American Health Organization; 2008. / citado 6 Set. 2014/. Disponible en: http://www2.paho.org/hq/

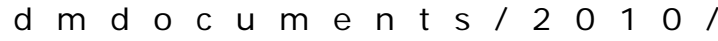
dengue_regional_data_2007.pdf

16.Instituto de Previsión Social. Departamento de Epidemiología. Informe técnico de situación de casos de dengue en IPS: Junio de 2007. Asunción: Instituto de Previsión Social; 2007.

17. Duarte A, López L, Iramain V, Álvarez E, Basualdo W, Alfieri P. Manejo del síndrome de shock dengue (SSD) en una UCIP. Pediatr. 2009; 36(1): 11-15.

18.Taboada A, Benítez G. Epidemiología, clínica y laboratorio de los pacientes con hepatitis y dengue [Resumen]. /citado 6 Set. 2014/. Disponible en: http://www.imt.edu.py/ a d m in / u p I o a d s / D o cumento/ hepatitisydengue.pdf

19.Gubler DJ. Dengue and dengue hemorrhagic fever. Clin Microbiol Reviews. 1998;11(3): 480-6.

20.World Health Organization. Dengue haemorrhagic fever: Diagnosis, treatment and control. Geneva: WHO; 1986.

21. Beatty M, Beutels P, Meltzer MI, Shepard DS, Hombach J, Hutubessy R, et al. Health economics of dengue: $A$ systematic literature review and expert panel's assessment. Am J Trop Med Hyg. 2011;84 (3): 473-88. 